\title{
Dynamic vortex mass in clean Fermi superfluids and superconductors
}

\author{
N. B. Kopnin ${ }^{(1,2)}$ and V. M. Vinokur ${ }^{(2)}$ \\ (1) L. D. Landau Institute for Theoretical Physics, 117940 Moscow, Russia \\ (2) Argonne National Laboratory, Argonne, Illinois 60439
}

(October 13, 2018)

\begin{abstract}
We calculate the dynamic vortex mass for clean Fermi superfluids including both $s$ - and $d$-wave superconductors as a response to a vortex acceleration. Assuming a finite quasiparticle mean free time, the vortex mass appears to be a tensor. The diagonal component dominates in the limit of long mean free time while the off-diagonal mass takes over in the moderately clean regime.
\end{abstract}

The vortex mass in superfluids and superconductors is a long standing problem in vortex physics and remains to be an issue of controversies. There are different approaches to its definition. One approach consists in calculating the vortex free energy increase due to the vortex velocity [1]. First used by Suhl [2] this approach yields the mass of the order of one quasiparticle mass (electron, in case of superconductor) per atomic layer (see Ref. [3] for an extensive review). Another approach is based on finding the force necessary to support an unsteady vortex motion. Identifying then the contribution to the force proportional to the vortex acceleration, one defines the vortex mass as a coefficient of proportionality. This method was first applied for vortices in super-

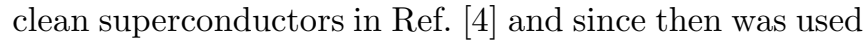
widely (see for example, [5] ). The resulting mass is of the order of the total mass of all particles within the area occupied by the vortex core. We will refer to this mass as to the dynamic mass. The dynamic mass originates from the inertia of excitations localized in the vortex core and can also be calculated as the momentum carried by localized excitations [8]. It is much larger and thus it is much more important than the mass obtained from the energy considerations.

In the present Letter we develop a regular microscopic approach for calculating the dynamic vortex mass in a general case of a finite relaxation time $\tau$ of nonequilibrium excitations produced by the moving vortex. Using the Boltzmann kinetic equation for quasiparticles localized in the vortex core, we derive the equation for the vortex dynamics which contains the inertia term together with all the forces acting on a moving vortex. This approach is applied to both $s$ - and $d$-wave superconductors. We find that dynamic mass displays a novel feature: it is a tensor whose components depend on the quasiparticle relaxation time. In $s$-wave superconductors, this tensor is diagonal in the superclean limit. The diagonal mass decreases rapidly as a function of the mean free time, and the off-diagonal components dominate in the moderately clean regime. In $d$-wave superconductors, the transition from a diagonal mass (in the extremely clean, high- $\tau$ limit) to an off-diagonal mass tensor (in the moderately clean, low- $\tau$ regime) occurs via the intermediate universal regime of the flux flow predicted in [9] (see also [10, 11]), where both components are of the same order of magnitude. Our results agree with the previous work 4, 5,8,11] in the limit $\tau \rightarrow \infty$.

Forces on a moving vortex. The Boltzmann kinetic equation for quasiparticles localized in the vortex core is

$$
\frac{\partial f}{\partial t}+\frac{\partial f}{\partial \alpha} \frac{\partial \epsilon_{n}}{\partial \mu}-\frac{\partial \epsilon_{n}}{\partial \alpha} \frac{\partial f}{\partial \mu}=\left(\frac{\partial f}{\partial t}\right)_{\text {coll }} .
$$

Here $\epsilon_{n}(\alpha, \mu)$ is the quasiclassical spectrum of a quasiparticle, which depends on canonically conjugated variables $\alpha$ and $\mu$ where $\alpha$ is the angle of the particle velocity $\hat{\mathbf{v}}_{\perp}= \pm \hat{\mathbf{p}}_{\perp}$ in the plane perpendicular to the vortex axis, and $\mu=\mp p_{\perp} b$ is the "angular momentum" defined through the impact parameter $b$ of a particle. The impact parameter is a good quantum number in the quasiclassical limit and so is $\mu$, even if there is no axial symmetry. The $\mp$ signs refer to particles and holes in the sense of the normal-state Fermi surface with positive and negative effective masses (in the plane perpendicular to the vortex axis), respectively. We take the coordinate $z$ axis along the vortex circulation $\hat{\mathbf{z}}=\operatorname{sgn}(e) \mathbf{B} / B$ where $\mathbf{B}$ is the magnetic induction. It can be shown 12 that, for the vortex dynamics, the Boltzmann-equation approach 13. is equivalent to the full-scale Green function formalism [14,15]. Eq. (11) can be derived for slow in time variations of the distribution function such that the characteristic $\omega$ is small as compared to $T$ or to the bulk gap $\Delta_{0}$.

For simplicity, we restrict ourselves to the case of well separated vortices, $H \ll H_{c 2}$, and consider the limit $\omega_{c} \tau \ll 1$ where $\omega_{c}=|e| H / m^{*} c$ is the cyclotron frequency. The limit $\omega_{c} \tau \ll 1$ is the most realistic for superconductors; it also corresponds to uncharged superfluids. In this limit, the delocalized quasiparticles above the gap are in equilibrium with the heat bath and do not participate in the vortex motion 15 .

We seek the distribution function in the form $f=$ $f^{(0)}\left(\epsilon_{n}\right)+f_{1}$, where $f^{(0)}(\epsilon)=1-2 n(\epsilon)$ and $n(\epsilon)$ is the Fermi function while $f_{1}$ describes the deviation from equilibrium. For a vortex moving with the velocity $\mathbf{u}$ the angular momentum is time dependent: $\mu(t)=$ $[(\mathbf{r}-\mathbf{u} t) \times \mathbf{p}] \cdot \hat{\mathbf{z}}$. The driving term in Eq. (11) is 


$$
\frac{\partial f^{(0)}}{\partial t}=\frac{\partial f^{(0)}}{\partial \epsilon} \frac{\partial \epsilon_{n}}{\partial \mu}\left(\left[\mathbf{p}_{\perp} \times \mathbf{u}\right] \hat{\mathbf{z}}\right) .
$$

The spectrum of bound states in the vortex core has an anomalous chiral branch [16] $\epsilon_{0}(\mu)$ whose energy varies from $-\Delta_{0}$ to $\Delta_{0}$ crossing zero as the impact parameter of a particle in the core varies from $-\infty$ to $+\infty$. The anomalous branch corresponds to the radial quantum number $n=0$. The energies of states with $n \neq 0$ are separated from $\epsilon=0$ by a gap of the order of $\Delta_{0}$. Defining $\omega_{n}=\mp \partial \epsilon_{n} / \partial \mu$, we ensure $\omega_{n}$ to be positive on the branch $n=0$ for both particles with $\hat{\mathbf{v}}_{\perp}=\hat{\mathbf{p}}_{\perp}$ and holes with $\hat{\mathbf{v}}_{\perp}=-\hat{\mathbf{p}}_{\perp}$. Note that it is the Fermi velocity $\mathbf{v}_{\perp}$ rather than the momentum $\mathbf{p}_{\perp}$ that enters the quasiclassical equations which determine the excitation spectrum. Consequently, it is $\partial \epsilon_{n} / \partial b$ that has the same sign for particles and holes.

Multiplying Eq. (1) by $\mathbf{p}_{\perp} / 2$ and summming up over all the quantum numbers, we obtain

$$
\mathbf{F}_{\text {env }}=\mathbf{F}_{\text {loc }}-\frac{\partial \mathbf{P}}{\partial t}-\pi\left(N_{e}-N_{h}\right) \tanh \left(\frac{\Delta_{0}}{2 T}\right)[\mathbf{u} \times \hat{\mathbf{z}}]
$$

where the 1.h.s. of Eq. (3) is the force from the environment on a moving vortex derived in [15]. It is the momentum transferred from excitations to the vortex:

$$
\mathbf{F}_{\mathrm{env}}=\frac{1}{2} \sum_{n} \int \frac{d p_{z}}{2 \pi} \frac{d \alpha d \mu}{2 \pi}\left[\hat{\mathbf{z}} \times \mathbf{p}_{\perp}\right] \frac{\partial \epsilon_{n}}{\partial \mu} f_{1} .
$$

The first term in the r.h.s. of Eq. (3) is the force exerted on the vortex by the heat bath via excitations localized in the vortex core:

$$
\mathbf{F}_{\text {loc }}=-\frac{1}{2} \sum_{n} \int \frac{d p_{z}}{2 \pi} \frac{d \alpha d \mu}{2 \pi} \mathbf{p}_{\perp}\left(\frac{\partial f}{\partial t}\right)_{\text {coll }} .
$$

Its component parallel to $\mathbf{u}$ gives the friction force. The transverse component is nothing but the spectral flow force due to the localized excitations [17. The second term in the r.h.s. of (3) is the change in the vortex momentum defined as

$$
\mathbf{P}=-\frac{1}{2} \sum_{n} \int \frac{d p_{z}}{2 \pi} \frac{d \alpha d \mu}{2 \pi} \mathbf{p}_{\perp} f_{1} .
$$

Finally, the third term in the r.h.s. of Eq. (3) is obtained from Eq. (2) (we assume here an $s$-wave pairing for simplicity). The sum over $n$ contains only the spectral branch with $n=0$ because $\partial \epsilon_{n} / \partial \mu$ is odd in $\mu$ for $n \neq 0$. It can be written as

$$
\begin{aligned}
& -\pi N_{s}[\mathbf{u} \times \hat{\mathbf{z}}]-\pi N_{n}[\mathbf{u} \times \hat{\mathbf{z}}] \\
& +\pi N\left[1-\tanh \left(\frac{\Delta_{0}}{2 T}\right)\right][\mathbf{u} \times \hat{\mathbf{z}}] .
\end{aligned}
$$

Here we put $N=N_{e}-N_{h}$ and $N_{s}=N-N_{n}$ where $N_{n}$ is the normal density. The term containing $N_{n}$ in Eq. (7) is the Iordanskii force [18] while the third one is a part of the spectral flow force due to the excitations above the gap [17]. The last two terms in Eq. (7) together can be written as the transverse force produced by the delocalized excitations scattered by the vortex potential

$$
\mathbf{F}_{\mathrm{sc} \perp}=-[\mathbf{u} \times \hat{\mathbf{z}}] \int_{|\epsilon|>\Delta_{0}} \frac{d \epsilon}{4} \frac{\partial f^{(0)}}{\partial \epsilon} \int \frac{d p_{z}}{2 \pi} p_{\perp}^{3} \sigma_{\perp}
$$

with the transverse cross section obtained in Ref. 19]

$$
\sigma_{\perp}=\frac{\pi}{p_{\perp}}\left(\frac{\epsilon}{\sqrt{\epsilon^{2}-\Delta_{0}^{2}}}-1\right) .
$$

Note that the similar longitudinal force can be neglected since it is smaller than the transverse one by a factor $\left(p_{F} \xi\right)^{-1}$ [19]. Eq. (3) becomes

$$
\mathbf{F}_{\mathrm{env}}=\mathbf{F}_{\mathrm{loc}}-\frac{\partial \mathbf{P}}{\partial t}-\pi N_{s}[\mathbf{u} \times \hat{\mathbf{z}}]+\mathbf{F}_{\mathrm{sc}} .
$$

The equation of the vortex dynamics is obtained by the variation of the superfluid free energy plus the energy in the external field with respect to the vortex displacement. The variation of the superfluid free energy gives the force from the environment, $\mathbf{F}_{\text {env }}$, while the variation of the energy in the external field produces the external force. For charged superfluids the external force is just the Lorentz force [23] $\mathbf{F}_{L}=\left(\Phi_{0} / c\right)\left[\mathbf{j}_{s} \times \hat{\mathbf{z}}\right] \operatorname{sgn}(e)$ where $\Phi_{0}=\pi c / 2|e|$ is the magnetic flux quantum. This formula holds also for uncharged systems since the charge drops out giving the external force in the form $\mathbf{F}_{L}=\pi N_{s}\left[\mathbf{v}_{s} \times \hat{\mathbf{z}}\right]$. In the absence of pinning, $\mathbf{F}_{L}+\mathbf{F}_{\text {env }}=0$ since the total energy is translationally invariant. Using Eq. (10) the force balance takes the form

$$
\mathbf{F}_{L}-\pi N_{s}[\mathbf{u} \times \hat{\mathbf{z}}]+\mathbf{F}_{\mathrm{loc}}+\mathbf{F}_{\mathrm{sc}}=\frac{\partial \mathbf{P}}{\partial t} .
$$

In a Galilean invariant system, where $N$ is the total number of carriers, the $N_{s}$-term can be combined with the Lorentz force to give the Magnus force $\mathbf{F}_{M}=$ $\pi N_{s}\left[\left(\mathbf{v}_{s}-\mathbf{u}\right) \times \hat{\mathbf{z}}\right]$. Eq. (11) becomes

$$
\mathbf{F}_{M}+\mathbf{F}_{\mathrm{loc}}+\mathbf{F}_{\mathrm{sc}}=\frac{\partial \mathbf{P}}{\partial t}
$$

The physical meaning of the Eq. (11) [or (12)] is simple. The equation accounts for all the forces acting on a moving straight vortex line: the Magnus force from the superfluid component, the force due to the scattering of normal excitations $\mathbf{F}_{\mathrm{sc}}$, and the force from the heat bath transferred to the vortex by localized excitations, $\mathbf{F}_{\text {loc }}$. The r.h.s. of Eqs. (11, 12) comes from the inertia of excitations localized in the vortex core and is identified as the change in the vortex momentum. The definition of Eq. (6) is similar to that used in Refs. [8, 13]. Note that the delocalized quasiparticles do not contribute to 
the vortex momentum in the limit $\omega_{c} \tau \ll 1$ because their distribution is equilibrium [15] with $f_{1}=0$.

Vortex mass. Having defined the vortex momentum, we calculate the vortex mass. The distribution function of localized particles is 15

$$
f_{1}=-\frac{d f^{(0)}}{d \epsilon}\left[\gamma_{\mathrm{H}}\left(\mathbf{u} \cdot \mathbf{p}_{\perp}\right) \pm \gamma_{\mathrm{O}}\left(\left[\mathbf{u} \times \mathbf{p}_{\perp}\right] \cdot \hat{\mathbf{z}}\right)\right]
$$

where $\gamma_{\mathrm{H}}$ and $\gamma_{\mathrm{O}}$ are to be found from Eq. (11). The vortex momentum becomes $P_{i}=M_{i k} u_{k}$; it has both longitudinal and transverse components with respect to the vortex velocity.

For a vortex with the symmetry not less than the fourfold, the effective mass tensor per unit length is $M_{i k}=$ $M_{\|} \delta_{i k}-M_{\perp} e_{i k j} \hat{z}_{j}$ where $M_{\|}=M_{\| e}+M_{\| h}$ and $M_{\perp}=$ $M_{\perp e}-M_{\perp h}$ with

$$
M_{\| e, h}=\frac{1}{4} \sum_{n} \int_{e, h} \frac{d p_{z}}{2 \pi} \frac{d \mu d \alpha}{2 \pi} \frac{d f^{(0)}}{d \epsilon} p_{\perp}^{2} \gamma_{\mathrm{H}}
$$

and the same expression for $M_{\perp e, h}$ with $\gamma_{\mathrm{H}}$ replaced with $\gamma_{\mathrm{O}}$. The $e, h$ subscripts indicate the integrations over the electron and hole parts of the Fermi surface, respectively. Only the branch with $n=0$ gives the contribution to the transverse mass because $\gamma_{\mathrm{O}}$ is odd in $\mu$ for $n \neq 0$.

Eq. (6) does not contain any explicit contribution from the so called "backflow mass". The backflow vortex mass was introduced 20] to account for an apparent similarity between a vortex core and an impenetrable cylinder immersed into a fluid. A hydrodynamic dipole counterflow would arise around the cylinder moving with respect to the fluid giving rise to the associated backflow mass 21]. We see, however, that the total inertial force comes from the change in the momentum of localized excitations only. In a sense, Eq. (6) takes care of all sources of the vortex mass including the possible backflow effect.

We also comment on arguments of Ref. [7] that the Coulomb interactions from charge variations produced by the moving vortex would screen the dynamic mass in charged superfluids. It is well known that moving vortex induces a non-equilibrium chemical potential which compensates the changes in the charge density (see, for example, 22,23]) . Therefore, the vortex motion does not violate the charge neutrality and the abovementioned screening effects are absent. (Note also that such a screening would first of all have screened out the whole vortex response to an electric current.)

$s$-wave superconductors. For $s$ wave superconductors, $\omega_{n}$ is independent of $\alpha$. If the collision integral is taken in the $\tau$-approximation $(\partial f / \partial t)_{\text {coll }}=-f_{1} / \tau_{n}$, the solution to Eq. (11) for a steady vortex motion is [15]

$$
\gamma_{\mathrm{H}}=\frac{\omega_{n}^{2} \tau_{n}^{2}}{\omega_{n}^{2} \tau_{n}^{2}+1} ; \gamma_{\mathrm{O}}=\frac{\omega_{n} \tau_{n}}{\omega_{n}^{2} \tau_{n}^{2}+1}
$$

where $\omega_{n} \sim T_{c}^{2} / E_{F}$. At low temperatures one has

$$
M_{\| e, h}=\pi N_{e, h}\left\langle\frac{\gamma_{\mathrm{H}}}{\omega_{0}}\right\rangle, M_{\perp e, h}=\pi N_{e, h}\left\langle\frac{\gamma_{\mathrm{O}}}{\omega_{0}}\right\rangle
$$

where $\langle\ldots\rangle=\left(\pi / V_{F}\right) \int p_{\perp}^{2} d p_{z}(\ldots)$ is the averaging over the Fermi surface with the volume $V_{F}$. The mass is of the order of the mass of electrons in the area occupied by the vortex core $M \sim \pi \xi_{0}^{2} m N$. In the superclean limit $T_{c}^{2} \tau / E_{F} \gg 1$ where $\omega_{n} \tau \gg 1$ the mass tensor is diagonal $M_{i k}=M_{\|} \delta_{i k}$, and we obtain the known result [4, 4,8$]$ : $M_{\| e, h}=\pi N_{e, h}\left\langle\omega_{0}^{-1}\right\rangle$.

The mass decreases with $\tau$. In the moderately clean regime $T_{c}^{2} \tau / E_{F} \ll 1$ where $\omega_{n} \tau \ll 1$, the diagonal component vanishes as $\tau^{2}$, and the mass tensor is dominated by the off-diagonal part. If $\tau_{0}$ is independent of energy

$$
M_{\perp e, h}=\pi \tanh \left(\frac{\Delta_{0}}{2 T}\right) N_{e, h}\left\langle\tau_{0}\right\rangle .
$$

The vortex mass is not a constant quantity for a given system: it may depend on the frequency $\omega$ of the external drive. Indeed, for $\omega$ comparable with $\omega_{0}$ and $\tau^{-1}$, both the forces and the effective mass acquire a frequency dispersion. From Eq. (11) one finds

$$
\gamma_{\mathrm{H}}=\frac{\omega_{n}^{2} \tau_{n}^{2}}{\omega_{n}^{2} \tau_{n}^{2}+\left(1-i \omega \tau_{n}\right)^{2}} ; \gamma_{\mathrm{O}}=\frac{\omega_{n} \tau_{n}\left(1-i \omega \tau_{n}\right)}{\omega_{n}^{2} \tau_{n}^{2}+\left(1-i \omega \tau_{n}\right)^{2}} .
$$

$d$-wave superconductors. We take the $d$-wave order parameter in the form $\Delta=\Delta_{0}(\rho, \phi) \sin (2 \alpha) e^{i \phi}$. The interlevel spacing $\omega_{n}(\alpha)$ for the quasiclassical spectrum $\epsilon_{n}(\alpha, \mu)$ of particles in the vortex core depends now on the momentum direction $\alpha$.

One distinguishes three different regimes for systems with different purity. In the order of increasing $\tau$ we have (i) the moderately clean regime, (ii) the universal regime, and (iii) the extremely clean limit. The moderately clean limit corresponds to $T_{c}^{2} \tau / E_{F} \ll 1$. In this case, the factors $\gamma$ in Eq. (13) are $\gamma_{\mathrm{H}}=\omega_{n}^{2}(\alpha) \tau_{n}^{2}$ and $\gamma_{\mathrm{O}}=\omega_{n}(\alpha) \tau_{n}$. The mass tensor has qualitatively the same behavior as for an $s$-wave superconductor.

In the superclean limit $T_{c}^{2} \tau / E_{F} \gg 1$, the specifics of a $d$-wave superconductor is more pronounced [9,11]. Consider temperatures $T \ll T_{c} \sqrt{H / H_{c 2}}$. At such temperatures, only localized states on the anomalous branch are excited. We need the quasiclassical spectrum for momentum directions near the gap nodes. The distance between the quasiclassical levels on the anomalous branch near the gap nodes is [11]

$$
\omega_{0}(\alpha)=8 \Omega_{0} \alpha^{2}+\omega_{c} / 2
$$

where $\Omega_{0} \sim T_{c}^{2} / E_{F}$, and $\omega_{c}=|e| H v_{\perp} / c p_{\perp}$ is the cyclotron frequency. For comparison, $\omega_{c} / \Omega_{0} \sim H / H_{c 2}$. The factors $\gamma_{\mathrm{H}}$ and $\gamma_{\mathrm{O}}$ in Eq. (13) satisfy the set of equations which follow from Eq. (1) 9, 11.

$$
\begin{aligned}
\frac{\partial \gamma_{\mathrm{O}}}{\partial \alpha}-\gamma_{\mathrm{H}}-U(\alpha) \gamma_{\mathrm{O}}+1 & =0, \\
\frac{\partial \gamma_{\mathrm{H}}}{\partial \alpha}+\gamma_{\mathrm{O}}-U(\alpha) \gamma_{\mathrm{H}} & =0 .
\end{aligned}
$$


Here $U(\alpha)=\left[\omega_{0}(\alpha) \tau_{0}\right]^{-1}$. The masses are

$$
\begin{aligned}
M_{\|, \perp} & =\int \frac{d p_{z}}{2 \pi} p_{\perp}^{2} \int_{-\pi / 4}^{\pi / 4} \frac{d \alpha}{2 \pi} \int \omega_{0}(\alpha) d \mu \frac{d f^{(0)}}{d \epsilon} \frac{\gamma_{\mathrm{H}, \mathrm{O}}(\alpha)}{\omega_{0}(\alpha)} \\
& =\frac{\tau}{\pi} \int \frac{d p_{z}}{2 \pi} p_{\perp}^{2} \int_{-\pi / 4}^{\pi / 4} d \alpha \frac{d F}{d \alpha} \gamma_{\mathrm{H}, \mathrm{O}}
\end{aligned}
$$

where $F(\alpha)=\int_{0}^{\alpha} U\left(\alpha^{\prime}\right) d \alpha^{\prime}$ and $\tau \equiv \tau_{0}(\alpha=0)$. The integral over $d \alpha$ is determined by the angles $\alpha \sim \sqrt{H / H_{c 2}}$. For this range, the terms with $U(\alpha)$ in Eq. (17) dominate and the factors $\gamma_{\mathrm{H}, \mathrm{O}}$ are 9,11

$$
\gamma_{\mathrm{H}}=\frac{\cosh \lambda e^{F(\alpha)}}{2 \sinh ^{2} \lambda+1}, \gamma_{\mathrm{O}}=\frac{\sinh \lambda e^{F(\alpha)}}{2 \sinh ^{2} \lambda+1} .
$$

Here

$$
\lambda=\int_{0}^{\infty} \frac{d \alpha^{\prime}}{\omega_{0}\left(\alpha^{\prime}\right) \tau}=\frac{\pi}{4 E_{0} \tau}
$$

and $E_{0}=\sqrt{\Omega_{0} \omega_{c}}$ is the distance between the exact quantum levels in the vortex core. They can be found by semi-classical quantization of the azimuthal motion of the particle having the spectrum $\epsilon_{0}(\alpha, \mu)$ [11. Finally, the masses become

$$
\begin{aligned}
& M_{\| e, h}=2 N_{e, h}\left\langle\frac{2 \tau \tanh \lambda}{\tanh ^{2} \lambda+1}\right\rangle, \\
& M_{\perp e, h}=2 N_{e, h}\left\langle\frac{2 \tau \tanh ^{2} \lambda}{\tanh ^{2} \lambda+1}\right\rangle .
\end{aligned}
$$

The universal regime [9] corresponds to $\lambda \gg 1$, i.e., $E_{0} \tau \ll 1$. In this limit, the spacing between the exact quantum levels is much smaller than the relaxation rate, while the average distance between the quasiclassical levels is larger than the relaxation rate, $\Omega_{0} \tau \gg 1$. Here, the both masses are of the same order of magnitude $M_{\|} \sim M_{\perp} \sim N \tau$. In the extremely clean limit when $\lambda \ll 1$, i.e., $E_{0} \tau \gg 1$, one obtains $M_{\| e, h}=\pi N_{e, h}\left\langle E_{0}^{-1}\right\rangle$ which agrees with the result of Ref. [8,11]. The transverse mass is small $M_{\perp} \sim \lambda M_{\|}$.

In conclusion, we have calculated the dynamic vortexmass tensor as a response to a slow vortex acceleration. The diagonal component dominates in the limit of a very long mean free time. In the opposite limit of fast relaxation, the off-diagonal mass is more important. The order of magnitude of the dynamic mass is the mass of all the quasiparticles in the area occupied by the vortex core. This mass is much larger than both the electrodynamic and the Suhl masses and is associated with the inertia of excitations localized in the vortex core.

We are grateful to G. Blatter, V. Geshkenbein, E. Sonin, and G. Volovik for stimulating discussions. This work was supported by the U.S. Department of Energy, BES-Materials Sciences, under contract \# W-31109-ENG-38 and by the NSF-Office of the Science and
Technology Center under contract No. DMR91-20000. NK also acknowledges support from the Russian Foundation for Fundamental Research grant No. 96-02-16072 and from the program "Statistical Physics" of the Ministry of Science of Russia and from the Swiss National Foundation cooperation grant 7SUP J048531.

[1] J.-M. Duan and A. J. Leggett, Phys. Rev. Lett. 68, 1216 (1992).

[2] H. Suhl, Phys. Rev. Lett. 14, 226 (1965).

[3] E. B. Sonin, V. B. Geshkenbein, A. van Otterlo, and G. Blatter, Phys. Rev. B 57, 575 (1998).

[4] N. B. Kopnin, Pis'ma Zh. Eksp. Teor. Fiz. 27, 417 (1978) [JETP Lett. 27, 390 (1978)].

[5] N. B. Kopnin and M. M. Salomaa, Phys. Rev. B, 44, 9667 (1991).

[6] E. Šimánek, Journ. Low Temp. Phys. 100, 1 (1995).

[7] D. M. Gaitonde and T. V. Ramakrishnan, Phys. Rev. B 56, 11951 (1997).

[8] G. E. Volovik, Pis'ma Zh. Eksp. Teor. Fiz. 65, 201 (1997) [JETP Lett. 65, 217 (1997)].

[9] N. B. Kopnin and G. E. Volovik, Phys. Rev. Lett. 79, 1377 (1997).

[10] Yu. G. Makhlin, Phys. Rev. B, 56,11 872 (1997).

[11] N. B. Kopnin, Phys. Rev. B 57, 11775 (1998).

[12] N. B. Kopnin, V. B. Geshkenbein, and G. Blatter, to be published.

[13] M. Stone, Phys. Rev. B 54, 13222 (1996).

[14] N. B. Kopnin and V. E. Kravtsov, Pis'ma Zh. Eksp. Teor. Fiz. 23, 631 (1976) [JETP Lett. 23, 578 (1976)].

[15] N. B. Kopnin and A. V. Lopatin, Phys. Rev. B 51, 15 291 (1995).

[16] C. Caroli, P. G. de Gennes, and J. Matricon, Phys. Lett. 9, 307 (1964).

[17] N. B. Kopnin, G. E. Volovik, and Ü. Parts, Europhys. Lett. 32, 651 (1995).

[18] S. V. Iordanskii, Ann. Phys. 29, 335 (1964).

[19] N. B. Kopnin and V. E. Kravtsov, Zh. Eksp. Teor. Fiz. 71, 1644 (1976) [Sov. Phys. JETP 44, 861 (1976)].

[20] G. Baym and E. Chandler, Journ. Low Temp. Phys. 50, 57 (1983).

[21] L. D. Landau and E. M. Lifshits, Fluid mechanics (Pergamon Press, N.Y. 1987).

[22] L. P. Gorkov and N. B. Kopnin, Usp. Fiz. Nauk 116, 413 (1975) [Sov. Phys. Uspekhi 18, 496 (1976)].

[23] A. I. Larkin and Yu. N. Ovchinnikov, in Nonequilibrium Superconductivity, edited by D. N. Langenberg and A. I. Larkin (Elsevier Science Publishers, 1986), p. 493. 